\title{
PERFORMANCE ASSESSMENT OF REAL-TIME MULTICONSTELLATION GNSS PPP USING A LOW-COST DUAL-FREQUENCY GNSS MODULE
}

\author{
Abdelsatar ELMEZAYEN, Ahmed EL-RABBANY \\ Department of Civil Engineering, Ryerson University, Toronto, Canada \\ e-mails: abdelsatar.elmezayen@,ryerson.ca, rabbany@,ryerson.ca
}

\begin{abstract}
The release of low-cost dual-frequency (DF) global navigation satellite system (GNSS) modules provides an opportunity for low-cost precise positioning to support autonomous vehicle applications. The new GNSS modules support the US global positioning system (GPS) L1C/L2C or L5 civilian signals, the Russian GNSS Globalnaya Navigazionnaya Sputnikovaya Sistema (GLONASS) L1/L2, Europe's GNSS Galileo E1/E5b, and Chinese GNSS BeiDou B1/B2 signals. The availability of the DF measurements allows for removal of the ionospheric delay, enhancing the obtained positioning accuracy. Unfortunately, however, the L2C signals are only transmitted by modernized GPS satellites. This means that fewer GPS DF measurements are available. This, in turn, might affect the accuracy and the convergence of the GPS-only precise point positioning (PPP) solution. Multi-constellation GNSS PPP has the potential to improve the positioning accuracy and solution convergence due to the high redundancy of GNSS measurements. This paper aims to assess the performance of real-time quad-constellation GNSS PPP using the low-cost u-blox Z9D-F9P module. The assessment is carried out for both open-sky and challenging environment scenarios. Static, simulated-kinematic, and actual field-kinematic trials have been carried out to evaluate real-time PPP performance. Pre-saved real-time precise orbit and clock products from the Centre National d'Etudes Spatiales are used to simulate the real-time scenario. It is shown that the quad-constellation GNSS PPP using the low-cost u-blox Z9DF9P module achieves decimeter-level positioning accuracy in both the static and simulatedkinematic modes. In addition, the PPP solution convergence is improved compared to the dual- and triple-constellation GNSS PPP counterparts. For the actual kinematic trial, decimeter-level horizontal positioning accuracy is achieved through the GPS + GLONASS + Galileo PPP compared with submeter-level positioning accuracy for the GPS + GLONASS and GPS + Galileo PPP counterparts. Additionally, submeter-level vertical positioning accuracy is achieved through the GPS + GLONASS + Galileo PPP compared with meter-level positioning accuracy for GPS + GLONASS and GPS + Galileo PPP counterparts.
\end{abstract}

Keywords: low-cost GNSS device, improved robust adaptive filter, real-time GNSS PPP

(C)The Author(s). This is an open-access article distributed under the terms of the Creative Commons Attribution License(CC BY 4.0, $\mathrm{https}$ ://creativecommons.org/licenses/by/4.0/), which permits use, distribution, and reproduction in any medium, provided that the Article is properly cited. 


\section{INTRODUCTION}

The precise point positioning (PPP) technique is attractive to the global navigation satellite system (GNSS) community due to its high precision without the need for additional base station infrastructure. Real-time precise GNSS satellite orbit and clock products have recently become available through the international GNSS service (IGS) and several analysis centers (Wang et al., 2018). This allows users to apply real-time global positioning system (GPS) PPP (Elsobeiey and Al-Harbi, 2016) or multi constellation GNSS PPP (Wang et al., 2018). Usually, a costly geodetic-grade GNSS receiver is required for real-time PPP to achieve precise positioning, which is an obstacle to applying real-time PPP for a wide range of commercial applications. To overcome this barrier, a number of researchers have adopted and used real-time single-frequency (SF)-PPP because of the low cost of SF GNSS chipsets and their adaptability for several applications (de Bakker and Tiberius, 2017, Elsheikh et al., 2018). The main challenge in real-time SF-PPP is eliminating ionospheric delay, as the quality of real-time ionosphere products significantly affects SF-PPP performance (Nie et al., 2019). In a previous paper (de Bakker and Tiberius, 2017), a u-blox M8T EVK SF GNSS device and a batch antenna were used for both static and kinematic experiments. IGS realtime orbit and clock products were also used. In addition, the predicted ionospheric maps from the Centre for Orbit Determination in Europe were utilized to account for the ionospheric delay. In that research, real-time SF GPS + Russian GNSS Globalnaya Navigazionnaya Sputnikovaya Sistema (GLONASS) PPP showed comparable results to the GPS-only counterpart in static and kinematic experiments. Moreover, the positioning performance was slightly improved through real-time SF GNSS PPP with a $30^{\circ}$ elevation angle in static and kinematic experiments (de Bakker and Tiberius, 2017).

Nie et al. (2019), using real-time SF-PPP, achieved submeter-level horizontal positioning accuracy and meter-level vertical positioning accuracy under an open-sky environment through the real-time ionosphere products of the Centre National d'Etudes Spatiales (CNES). The release of dual-frequency (DF) GNSS smartphones, such as the Xiaomi Mi 8, which supports DF measurements for GPS and Europe's GNSS Galileo satellite systems, allows users to remove the ionospheric delay, which in turn leads to an enhanced PPP solution. However, the quality of the smartphone's observations is relatively lower than those obtained from geodetic-grade GNSS receivers, and its exact antenna location is unknown. As a result, DF PPP through smartphones can achieve decimeter-to-submeter-level static positioning accuracy (Elmezayen and El-Rabbany, 2019, Psychas et al., 2019); however, this is not better than 2-m kinematic positioning accuracy (Wu et al., 2019).

Low-cost DF GNSS modules have recently become available, including the u-blox ZED-F9P module (u-blox). DF measurements enable ionosphere-free (IF) linear combinations, removing the ionospheric delay. This, in turn, increases the ability to provide high positioning accuracy with low-cost GNSS modules. The u-blox ZED-F9P module has been examined for zenith total tropospheric delay estimation (Krietemeyer et al., 2020) and ionosphere monitoring (Dan et al., 2020). This GNSS module supports only the civil signals L1 C/A and L2C for the GPS constellation. As of January 2021, the GPS constellation consists of the following: eight satellites of Block IIR and two of Block III, which can transmit one civil signal (L1 C/A); six satellites of Block IIR-M, which can transmit two civil signals (L1 C/A and L2C); and 12 satellites of Block IIF, which can transmit three civil signals (L1 C/A, L2C, and L5) (US Coast Guard). As a result, few DF measurements from the GPS are available to form the conventional IF linear combination. This may affect the resulting positioning accuracy, especially for kinematic applications in which all visible satellites need to be included in the positioning computation. In a previous paper (Nie et al., 2020), real-time DF low-cost GPS + GLONASS PPP was developed using the u-blox ZED-F9P along with CNES 
real-time products. The research focused on improving positioning accuracy and convergence behavior by combining both IF linear combination of DF measurements from GPS satellites that support the L2C signal and the ionosphere-corrected SF code measurements from satellites that support only L1 C/A. The CNES real-time ionosphere products were used to account for the ionospheric delay in the SF code measurements. The results showed that the proposed real-time DF-PPP solution is comparable to the SF-PPP for the first 3 minutes of the static experiment, but the DF-PPP accuracy was improved over time compared to the SFPPP. For the kinematic experiment, the proposed DF PPP showed similar convergence behavior as the real-time SF-PPP (Nie et al., 2020). The DF-PPP achieved decimeter-level horizontal and vertical positioning accuracy levels under an open-sky environment. As mentioned previously, the CNES real-time ionosphere products were used to mitigate the ionospheric delay of the SF code observations, which only can achieve positioning accuracy at the decimeter-to-submeter level (Nie et al, 2019). As a result, adding SF measurements may degrade the resulting positioning accuracy with low-quality ionosphere products. Moreover, the contribution of SF code measurements can be decreased or ignored under extreme ionospheric conditions due to the high uncertainty of ionospheric corrections (Nie et al., 2020).

In the present study, improvements in the positioning solution accuracy and convergence time are thoroughly analyzed using measurements from a quad-constellation, namely, GPS, GLONASS, Galileo, and Chinese GNSS BeiDou. An improved robust adaptive Kalman filter (IRKF) is adopted and used to remove the measurement outliers and compensate for the errors in the dynamic model, enhancing both the positioning solution accuracy and the convergence time. To validate the PPP algorithm's effectiveness, field trials in static, simulated-kinematic, and kinematic modes are conducted. The mathematical models for real-time multi constellation GNSS PPP and IRKF algorithms are first described, followed by a presentation of field trials and an analysis of the results. Concluding remarks are then summarized in the final section.

\section{MULTI CONSTELLATION GNSS PPP MATHEMATICAL MODELS}

In this work, GPS L1/L2, GLONASS L1/L2, Galileo E1/E5b, and BeiDou B1/B2 pseudorange and carrier-phase measurements are used to form the IF linear combinations. The measurement types used for the four constellations are presented in Table 1.

Table 1. GPS, GLONASS, Galileo, and BeiDou pseudorange and carrier-phase measurements

\begin{tabular}{|c|c|c|c|c|}
\hline Receiver type & GPS & GLONASS & Galileo & BeiDou \\
\hline U-blox-F9P & $\begin{array}{c}\text { C1C and L1C; } \\
\text { C2L and L2L }\end{array}$ & $\begin{array}{c}\text { C1C and L1C; } \\
\text { C2C and L2C }\end{array}$ & $\begin{array}{c}\text { C1C and L1C; } \\
\text { C7Q and L7Q }\end{array}$ & $\begin{array}{c}\text { C2I and L2I; } \\
\text { C7I and L7I }\end{array}$ \\
\hline
\end{tabular}

The IF linear combinations of the four constellations' pseudorange and carrier-phase measurements are applied as follows:

$$
\begin{gathered}
P_{I F}^{G}=\rho^{G}+b_{r}^{G}+m \underset{w}{G} z w d+\varepsilon_{P I F}^{G} \\
\Phi_{I F}^{G}=\rho^{G}+b_{r}^{G}+m_{w}^{G} z w d+N_{I F}^{G}+\varepsilon_{\Phi I F}^{G}
\end{gathered}
$$




$$
\begin{aligned}
& P_{I F}^{R}=\rho^{R}+b_{r}^{G}+m_{w}^{R} z w d+I S B^{R}+\varepsilon_{P I F}^{R} \\
& \Phi_{I F}^{R}=\rho^{R}+b_{r}^{G}+m_{w}^{R} z w d+N_{I F}^{R}+I S B^{R}+\varepsilon_{\Phi I F}^{R} \\
& P_{I F}^{E}=\rho^{E}+b_{r}^{G}+m \underset{w}{E} z w d+I S B^{E}+\varepsilon_{P I F}^{E} \\
& \Phi_{I F}^{E}=\rho^{E}+b_{r}^{G}+m_{w}^{E} z w d+N_{I F}^{E}+I S B^{E}+\varepsilon_{\Phi I F}^{E} \\
& P_{I F}^{C}=\rho^{C}+b_{r}^{G}+m \underset{w}{C} z w d+I S B B^{C}+\varepsilon_{P I F}^{C} \\
& \Phi_{I F}^{C}=\rho^{C}+b_{r}^{G}+m \underset{w}{C} z w d+N_{I F}^{C}+I S B B^{C}+\varepsilon_{\Phi I F}^{C}
\end{aligned}
$$

where:

$G, R, E$, and $C$ - GPS, GLONASS, Galileo, and BeiDou satellite systems, respectively;

$P_{I F}^{G}, \Phi_{I F}^{G}, P_{I F}^{R}, \Phi_{I F}^{R}, P_{I F}^{E}, \Phi_{I F}^{E} P_{I F}^{C}$, and $\Phi_{I F}^{C}-$ GPS, GLONASS, Galileo, and BeiDou IF linear combinations after accounting for the dry tropospheric delay, relativity, Sagnac delay, phase center offset and variation, Earth tides, ocean loading, and phase wind up errors;

$\rho^{G}, \rho^{R}, \rho^{E}$, and $\rho^{C}-$ geometric range between the receiver's antenna phase center and the corresponding satellite antenna phase center;

$$
\begin{array}{ll}
b_{r}^{G}=c \times d t_{r}^{G} & - \text { receiver clock error; } \\
z w d & - \text { the zenith wet delay; } \\
m_{w} & - \text { the wet mapping function; }
\end{array}
$$

$N_{I F}^{G}, N_{I F}^{R}, N_{I F}^{E}$, and $N_{I F}^{C}$ - the GPS, GLONASS, Galileo, and BeiDou IF ambiguity terms (in meters);

$I S B^{R}, I S B^{E}$, and $I S B^{C} \quad-$ the intersystem biases (ISBs) for the GLONASS, Galileo, and BeiDou satellite systems;

$\varepsilon_{P}, \varepsilon_{\varphi} \quad-$ the noise and multipath effect for the pseudorange and carrierphase measurements.

In the adopted GNSS PPP model, the pre-saved real-time orbit and clock corrections for GPS, GLONASS, Galileo, and BeiDou are obtained from the CNES analysis center (http:/www.ppp-wizard.net/products/REAL_TIME/). The code biases obtained from the CNES analysis center are applied to the GNSS pseudorange measurements to make them consistent with the satellite clock products. The tropospheric delay's dry zenith component is accounted for using the Saastamoinen model (Saastamoinen, 1973), while the dry and wet mapping functions are determined using the Vienna mapping function (VMF) (Böhm et al., 2015). The effects of relativity, Sagnac delay, phase center offset and variation, Earth tides, ocean loading, and phase wind-up are modeled as described by Kouba (2015).

\section{METHODOLOGY}

An IRKF is used in this research to reduce the effect of the measurement outliers and the dynamic model error on the obtained positioning accuracy. The modified measurement 
covariance matrix is constructed for the code and carrier-phase measurements of all four constellations. The IRKF is implemented in two steps, namely, the prediction and update of the state vector estimation:

Step 1: The predicted state vector and its covariance matrix are estimated as follows:

$$
\begin{gathered}
\delta x_{k}(-)=\Phi_{k, k-1} \delta x_{k-1}(+) \\
P_{k}(-)=\Phi_{k, k-1} P_{k-1}(+) \Phi_{k, k-1}^{T}+Q_{k, k-1}
\end{gathered}
$$

where:

$\delta x \quad-$ state vector, which includes position errors, receiver clock error, zenith wet tropospheric delay, ISB for $\mathrm{R}, \mathrm{E}$, and $\mathrm{C}$ satellite systems, and ambiguity;

$(-) \quad-$ refers to the predicted state;

$(+) \quad-$ refers to the updated state;

$k$ and $k-1 \quad-$ any two subsequent epochs;

$\Phi \quad-\quad$ transition matrix, which is the identity matrix;

$P \quad-$ state vector covariance matrix;

$Q \quad-$ covariance matrix of the process noise.

Step 2: An adaptive factor $\alpha$ is determined to balance the contribution between the predicted state vector and the estimated counterpart using the new measurements, as detailed by Xu and $\mathrm{Xu}(2016)$ :

$$
\alpha=\left\{\begin{array}{cc}
1 & \text { if }\left|\Delta \hat{X}_{k}\right| \leq c_{\circ} \\
\frac{c_{\circ}}{\left|\Delta \hat{X}_{k}\right|}\left(\frac{c_{1}-\left|\Delta \hat{X}_{k}\right|}{c_{1}-c_{\circ}}\right)^{2} & \text { if } c_{\circ}<\left|\Delta \hat{X}_{k}\right| \leq c_{1} \\
0 & \text { if }\left|\Delta \hat{X}_{k}\right|>c_{1}
\end{array}\right.
$$

where:

$c_{\circ}$ and $c_{1}-$ empirical values, which are chosen as $c_{\circ}=1.5, c_{1}=4.5$;

$\Delta \hat{X}_{k} \quad-$ the bias statistic and can be determined as follows:

$$
\Delta \hat{X}_{k}=\frac{\left\|\delta x_{k}(-)-\delta \widehat{x}_{k}\right\|}{\sqrt{\operatorname{tr}\left(P_{k}(-)\right)}}
$$

where:

$$
\begin{array}{ll}
\delta \hat{x}_{k} & - \text { the state vector estimate using the new measurements; } \\
t r & - \text { the matrix trace. }
\end{array}
$$

The higher the value of $\Delta \hat{X}_{k}$, the lower the value of the adaptive factor $\alpha$ leading to lower contribution from the predicted state vector.

Step 3: The Kalman gain $K$ and the updated state vector covariance matrix are then estimated as follows: 


$$
\begin{gathered}
K_{k}=\frac{1}{\alpha} P_{k}(-) H_{k}^{T}\left[\frac{1}{\alpha} H_{k} P_{k}(-) H_{k}^{T}+R_{k}\right]-1 \\
P_{k}(+)=\left(I-K_{k} H_{k}\right) P_{k}(-)\left(I-K_{k} H_{k}\right)^{T}+K_{k} R_{k} K_{k}^{T}
\end{gathered}
$$

Step 4: The standardized residuals are estimated for the code and carrier-phase measurements of all four constellations. A robust classification factor $F$ is then determined for each specific measurement type individually. The classification factor for the $i^{\text {th }}$ number of measurements in the $j^{\text {th }}$ type of measurements, $F_{i}^{j}$, can be estimated using the $t$-test statistics, as shown elsewhere (Zhang et al., 2018):

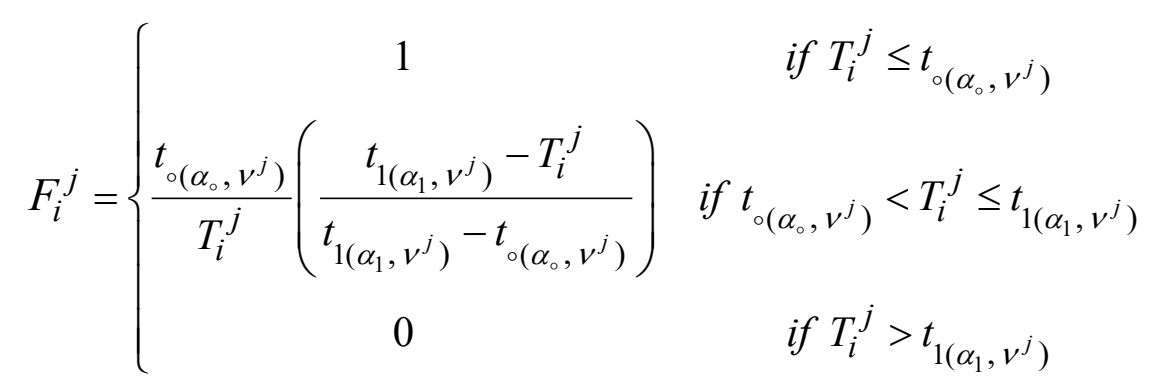

where:

$$
\begin{aligned}
& T_{i}^{j} \quad-\text { the } t \text {-test statistic for the } i^{\text {th }} \text { measurement in the } j^{\text {th }} \text { type, as described by } \\
& \text { Zhang et al. (2018); } \\
& t_{\circ}, t_{1}-\text { the } t \text {-test critical values at } \alpha_{\circ} \text { and } \alpha_{1} \text { significance levels; } \\
& v^{j=n^{j}-1}-\text { the degree of freedom; } \\
& n^{j}-\text { number of } j^{\text {th }} \text { type measurements; and significance levels are chosen as } \\
& \quad \alpha_{\circ}=0.15 \text { and } \alpha_{1}=0.01 .
\end{aligned}
$$

The elements of the modified measurement covariance matrix $\bar{R}$ can be constructed as follows:

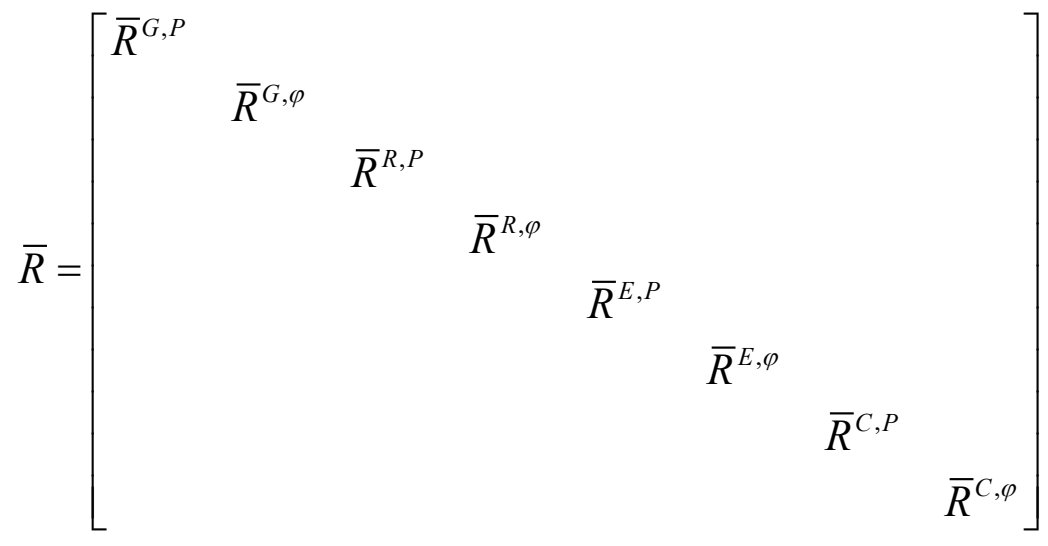

where:

$\bar{R}^{P}$ and $\bar{R}^{\varphi} \quad-$ the covariance matrices for the pseudorange and carrier-phase measurements for each satellite system,

$G, R, E$, and $C$ - the GPS, GLONASS, Galileo, and BeiDou satellite systems. 
$\bar{R}$ is determined based on both the corresponding elements of the original covariance matrix $R$ and the classification factors as follows:

$$
\bar{R}_{i}^{j}=R_{i}^{j} / F_{i}^{j}
$$

Step 5: The Kalman gain and the updated state covariance matrix can then be determined using the modified measurement covariance matrix $\bar{R}$ as follows:

$$
\begin{gathered}
\widehat{K}_{k}=\frac{1}{\alpha} P_{k}(-) H_{k}^{T}\left[\frac{1}{\alpha} H_{k} P_{k}(-) H_{k}^{T}+\bar{R}_{k}\right]-1 \\
\widehat{P}_{k}(+)=\left(I-\widehat{K}_{k} H_{k}\right) P_{k}(-)\left(I-\widehat{K}_{k} H_{k}\right)^{T}+\widehat{K}_{k} \bar{R}_{k} \widehat{K}_{k}^{T}
\end{gathered}
$$

Step 6: The updated state vector can be determined as follows:

$$
\delta x_{k}(+)=\delta x_{k}(-)+\hat{K}_{k}\left[\delta z_{k}-H_{k} \delta x_{k}(-)\right]
$$

where:

$\delta z-$ consists of the differences between the IF linear combinations GNSS

\begin{tabular}{|c|c|}
\hline Constellations & GPS, GLONASS, Galileo, and BeiDou \\
\hline Observations used & IF linear combinations of pseudorange and carrier phase \\
\hline Elevation mask angle & $7.5^{\circ}$ \\
\hline Observations weighting & Elevation dependent $[1 / \sin (\varepsilon)]$ \\
\hline Estimation filters & IRKF \\
\hline Satellites' orbit and clock & CNES real-time products \\
\hline $\begin{array}{l}\text { Satellite antenna phase } \\
\text { center off set and variation }\end{array}$ & Corrected (Rizos et al., 2013) \\
\hline Tropospheric delay & $\begin{array}{l}\text { Dry: applied using Saastamoinen model along with VMF mapping } \\
\text { functions; } \\
\text { Wet: estimated, modeled as a random walk process with spectral } \\
\text { density } 10^{-9} \mathrm{~m}^{2} / \mathrm{s}\end{array}$ \\
\hline Receiver clock error & $\begin{array}{l}\text { Estimated, modeled as a random walk process } \\
\text { with spectral density } 10^{5} \mathrm{~m}^{2} / \mathrm{s}\end{array}$ \\
\hline$I S B^{R}, I S B^{E}$, and $I S B^{B}$ & $\begin{array}{l}\text { Estimated, modeled as random walk processes } \\
\text { with spectral density } 10^{-7} \mathrm{~m}^{2} / \mathrm{s}\end{array}$ \\
\hline Ambiguities & Estimated (float values), modeled as random constants \\
\hline
\end{tabular}
measurements and the predicted counterparts. The full PPP processing details are summarized in Table 2.

Table 2. PPP processing details 


\begin{tabular}{|l|l|}
\hline Site displacements & $\begin{array}{l}\text { Solid Earth tides and ocean loading were applied } \\
\text { as in the paper by Kouba (2015) }\end{array}$ \\
\hline Relativity effect & Applied as in the paper by Kouba (2015) \\
\hline Phase wind-up & Applied as in the paper by Kouba (2015) \\
\hline
\end{tabular}

\section{EXPERIMENTAL SETUP AND ANALYSIS OF RESULTS}

Real-time multi constellation GNSS PPP through the IRKF was assessed in static, simulatedkinematic, and actual kinematic modes. A u-blox ZED-F9P GNSS module was connected to a u-blox patch antenna, and GNSS measurements were collected at the rate of $1 \mathrm{~Hz}$. The BeiDou satellite system was excluded from the actual kinematic processing due to the low number of visible BeiDou satellites during the data collection period. CNES pre-saved realtime orbit and clock products were used to simulate the real-time scenario. The IRKF was used as the estimation filter, as described in the "Methodology" section. The collected measurements were processed in different modes, namely, real-time GPS + GLONASS PPP (GR-PPP), GPS + Galileo PPP (GE-PPP), GPS + GLONASS + Galileo PPP (GRE-PPP), GPS + GLONASS + BeiDou PPP (GRC-PPP), and GPS + GLONASS + Galileo + BeiDou PPP (GREC) modes. To provide a reference solution, NovAtel's waypoint inertial explorer (IE) software was used to process the GNSS measurements in the carrier-phase-based differential GNSS (DGNSS) mode.

\subsection{Static field trial}

A field trial was conducted in a suburban area with an open-sky environment on June 20, 2020, and this process lasted for about 60 minutes. Figure 1 shows the visibility of the visible GNSS satellites during the whole data collection period. The minimum number of satellites was five, the maximum number was 25 , and the elevation mask angle was $7.5^{\circ}$. Figures $2-4$ present the positioning errors of the real-time GR-PPP, GE-PPP, GRC-PPP, GRE-PPP, and GREC-PPP solutions through the IRKF in the east, north, and up directions, respectively. The convergence performance of the GREC-PPP solution is similar to that of the GRC-PPP solution in the east and north directions and similar to the GRE-PPP solution in the up direction. The GE-PPP solution shows the worst performance, especially in the east direction. After about 30 minutes, the performances of the GR-PPP, GE-PPP, GRC-PPP, GRE-PPP, and GREC-PPP solutions tend to be similar in all positioning components, except for the east component, where the GE-PPP shows the worst performance. 


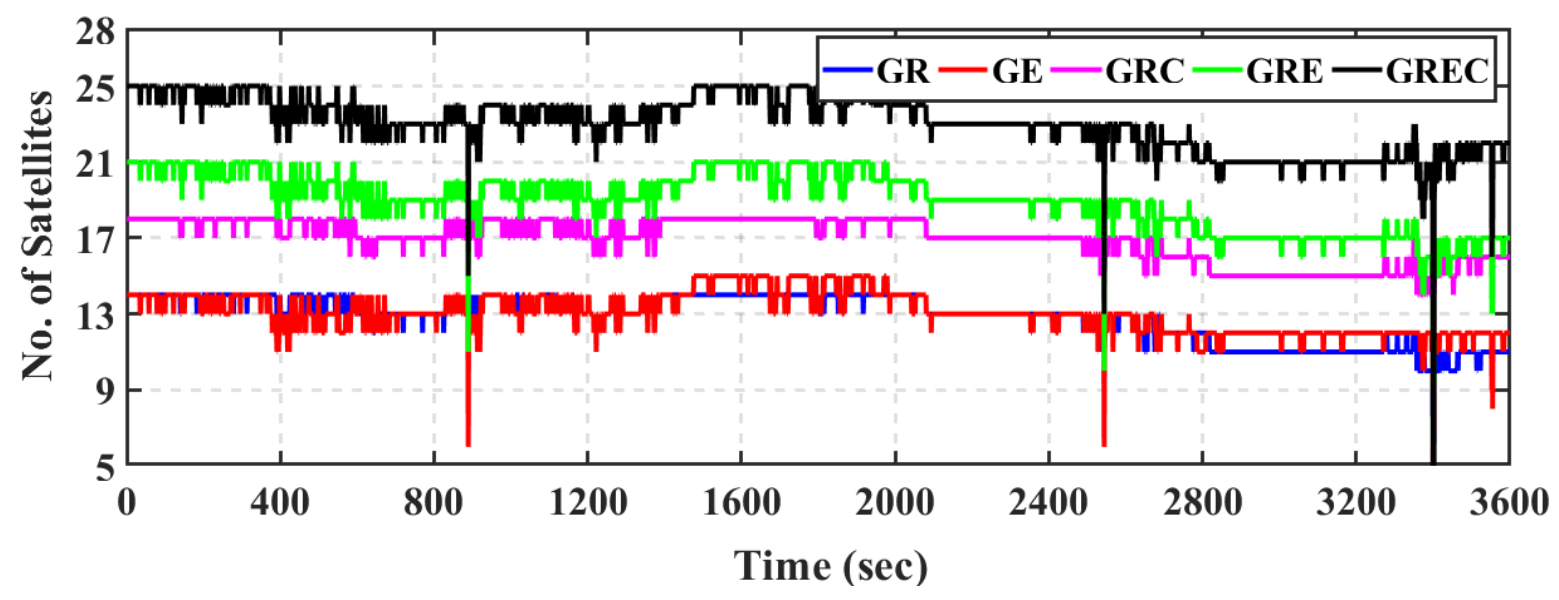

Figure 1. Static field trial: number of observable GPS + GLONASS, GPS + Galileo, GPS + GLONASS + BeiDou, GPS + GLONASS + Galileo, and GPS + GLONASS + Galileo + BeiDou satellites

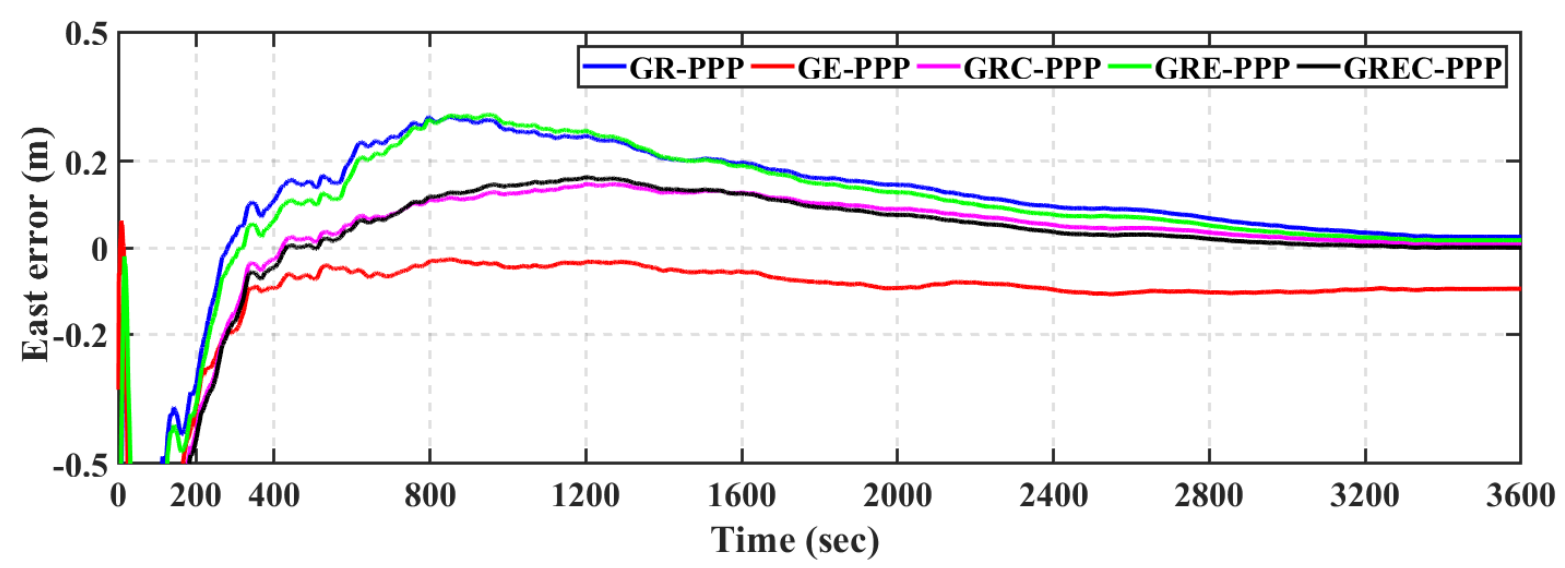

Figure 2. Static field trial: east positioning error of real-time GR-PPP, GE-PPP, GRE-PPP, GRC-PPP, and GREC-PPP solutions

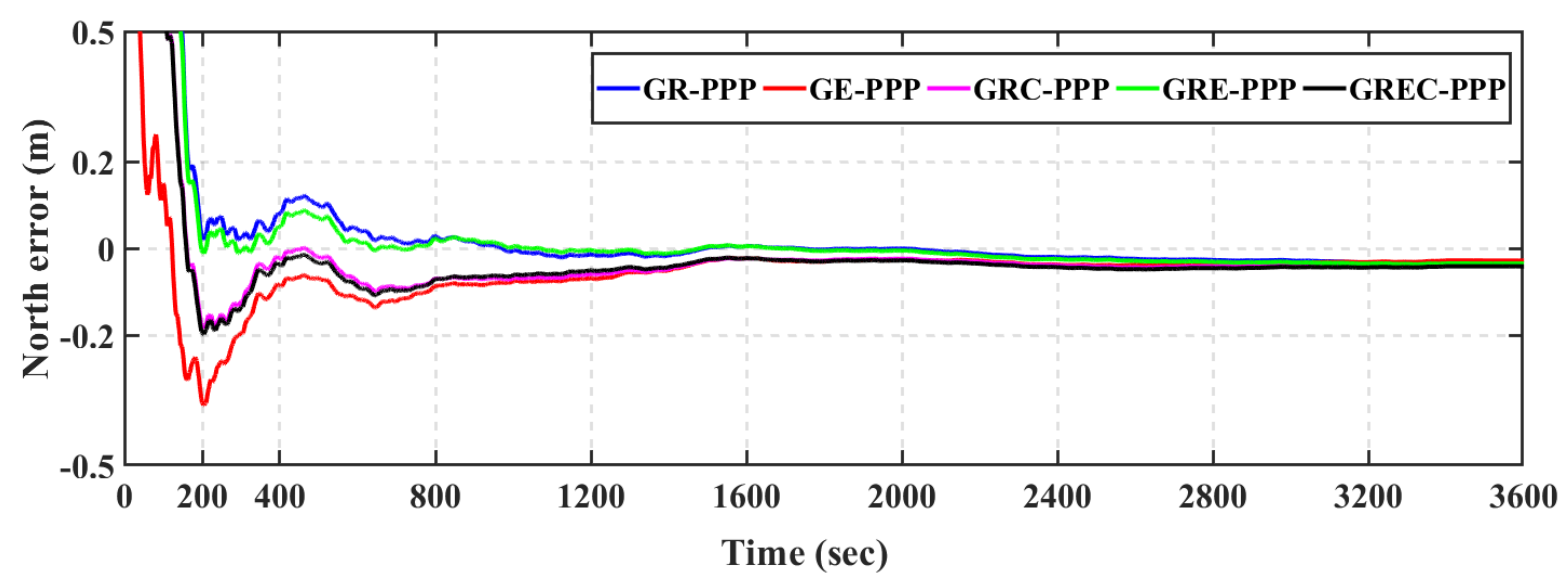

Figure 3. Static field trial: north positioning error of real-time GR-PPP, GE-PPP, GRE-PPP, GRC-PPP, and GREC-PPP solutions 


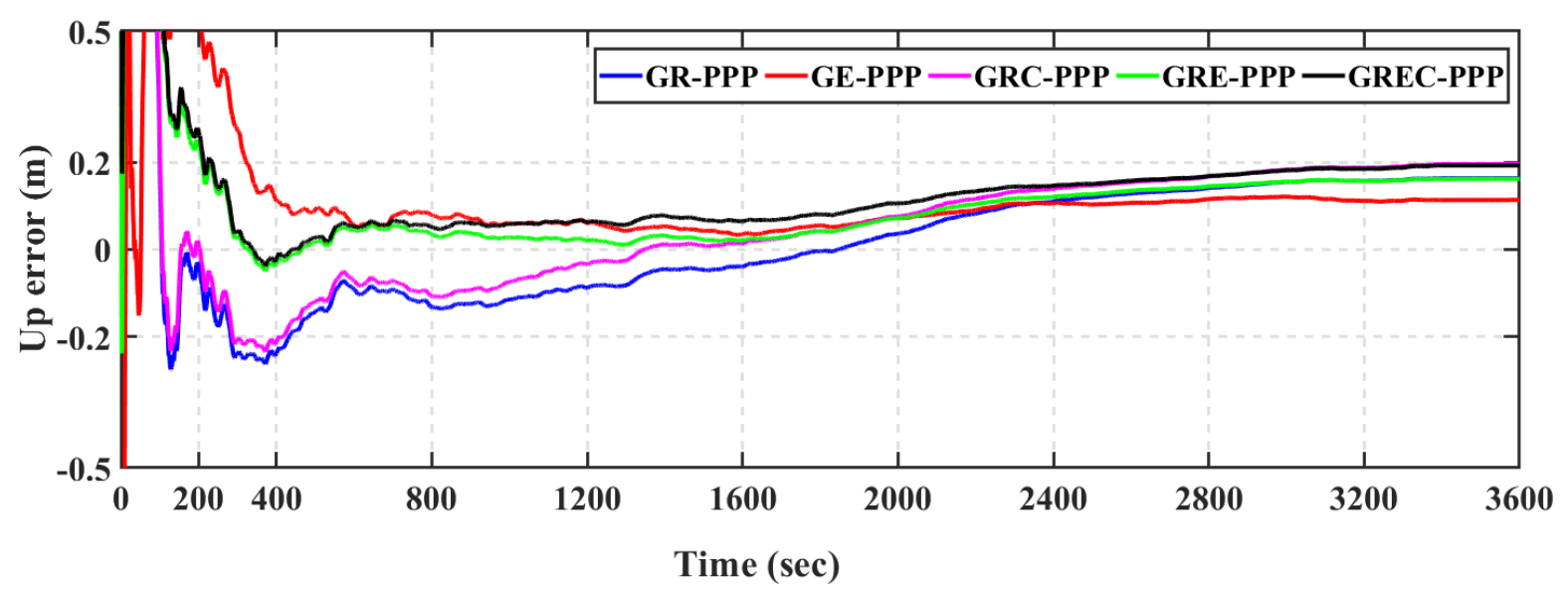

Figure 4. Static field trial: up positioning error of real-time GR-PPP, GE-PPP, GRE-PPP, GRC-PPP, and GREC-PPP solutions

To better assess the performance of the different positioning modes, the root-mean-square (RMS) error for each positioning component is calculated for different time windows of 10 minutes, 20 minutes, 30 minutes, 40 minutes, 50 minutes, and 60 minutes, as shown in Figures 5-7. As can be seen, the north direction's positioning accuracy is generally better than that of the east and up directions. For the same time window, the quad-constellation GNSS PPP (GREC-PPP) solution improves the positioning accuracy in comparison with the tripleconstellation GNSS PPP (GRE-PPP and GRC-PPP) solutions and the dual-constellation GNSS PPP (GE-PPP and GR-PPP) solutions for all positioning components. For example, a $20-\mathrm{cm}$ accuracy level is obtained within 20 minutes in the east and up directions and within 10 minutes in the north direction for the GREC-PPP solution. On the other hand, the GRPPP, GE-PPP, GRE-PPP, and GRC-PPP solutions achieve 20-cm accuracy within 40 minutes in the east and north directions and within 30 minutes in the up direction.

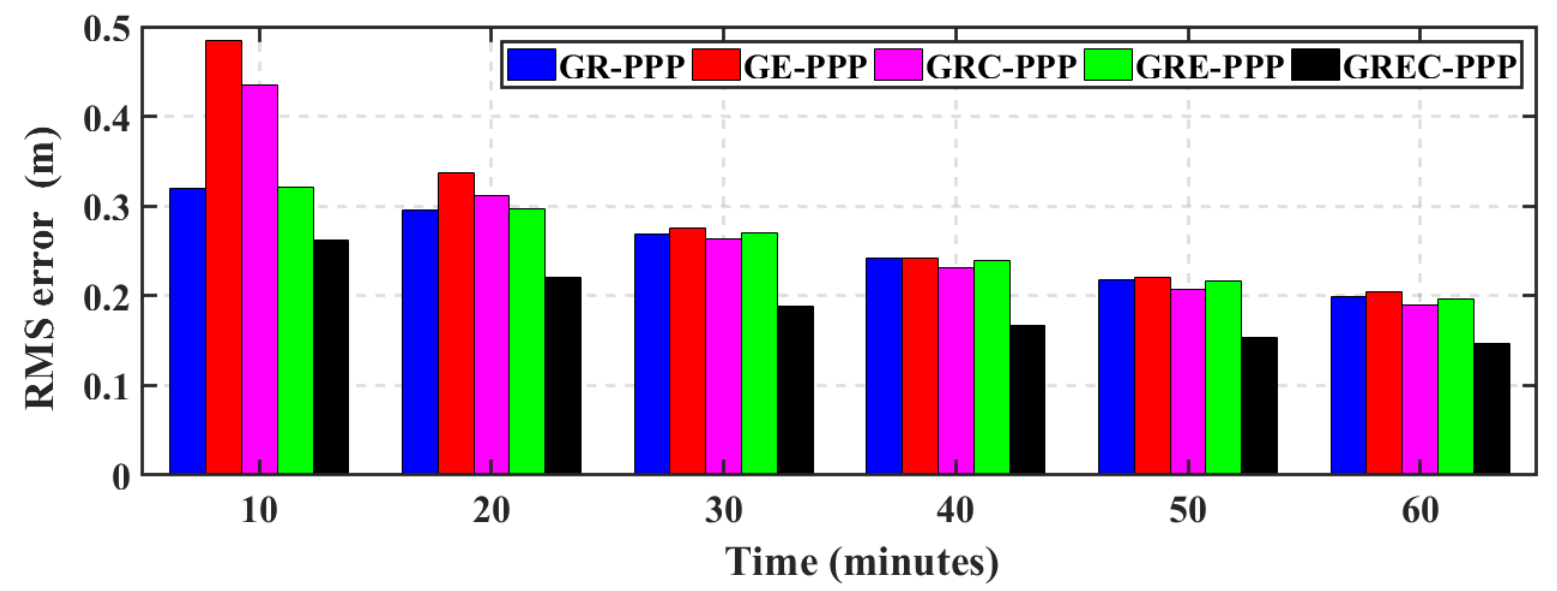

Figure 5. Static field trial: RMS error $(m)$ in the east direction of real-time GR-PPP, GE-PPP, GRE-PPP, GRC-PPP, and GREC-PPP solutions for different time windows 


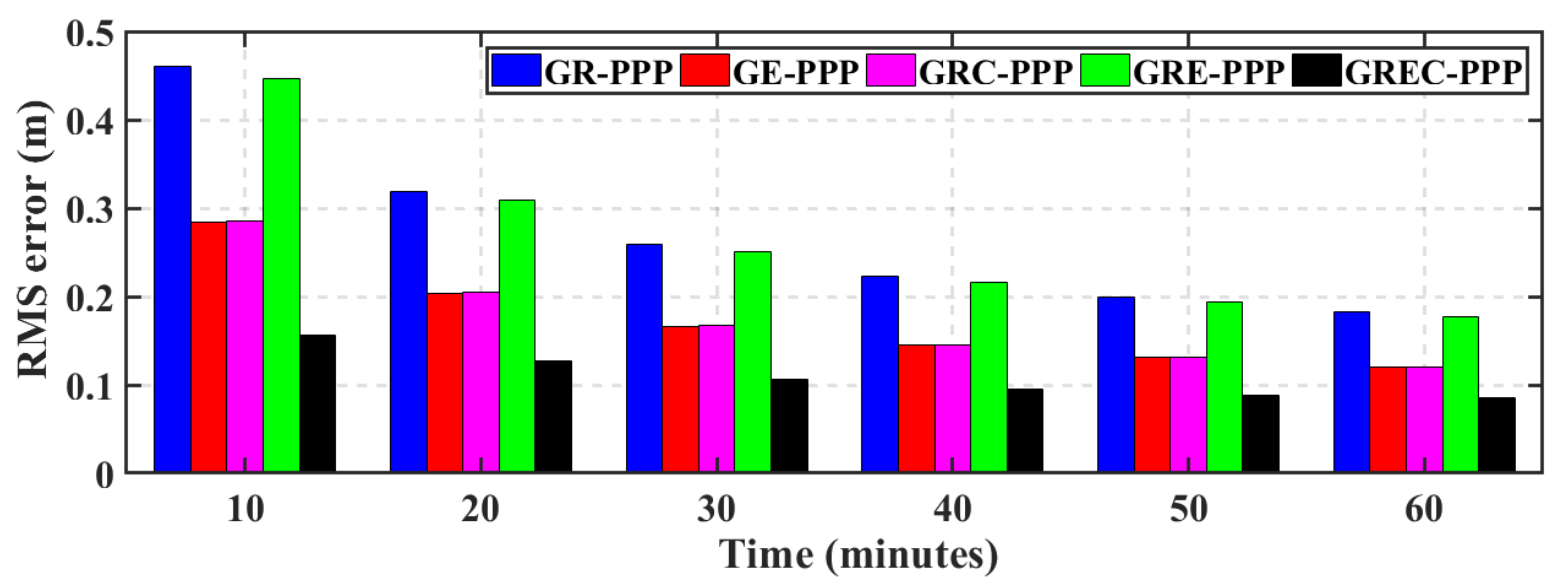

Figure 6. Static field trial: RMS error $(\mathrm{m})$ in the north direction of real-time GR-PPP, GE-PPP, and GRE-PPP, GRC-PPP, and GREC-PPP solutions for different time windows

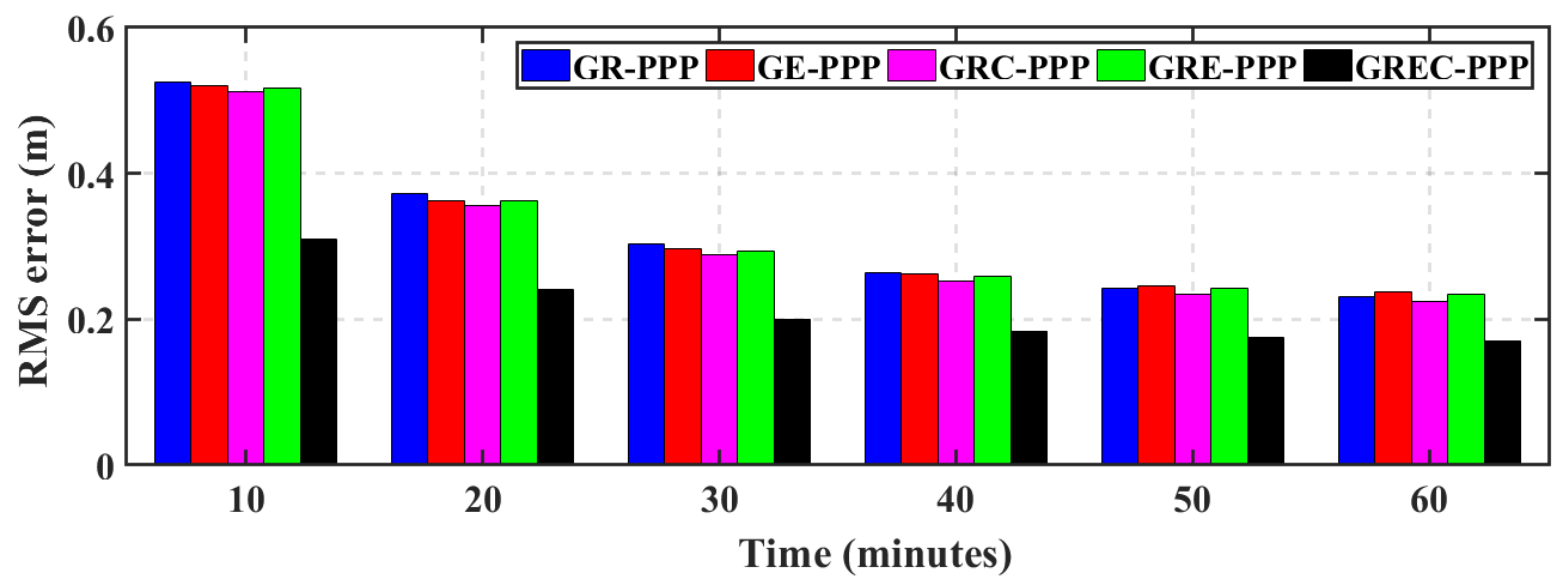

Figure 7. Static field trial: RMS error $(\mathrm{m})$ in the up direction of real-time GR-PPP, GE-PPP, GREPPP, GRC-PPP, and GREC-PPP solutions for different time windows

The RMS error and the mean of the GR-PPP, GE-PPP, GRE-PPP, GRC-PPP, and GRECPPP solutions in the east, north, and up directions are presented in Table 3. The GREC-PPP solution shows the best positioning accuracy for all positioning components, which coincides with the results from Figures 5-7. The accuracy of the GR-PPP solution is improved from $0.199 \mathrm{~m}, 0.183 \mathrm{~m}$, and $0.231 \mathrm{~m}$ in the east, north, and up directions, respectively, to $0.197 \mathrm{~m}$, $0.177 \mathrm{~m}$, and $0.234 \mathrm{~m}$ for the GRE-PPP solution. The GE-PPP and GRC-PPP solutions show similar levels of positioning accuracy in the north and up directions, which coincides with the results of Figures 6 and 7. The RMS of the GE-PPP positioning errors is $0.121 \mathrm{~m}$ and $0.237 \mathrm{~m}$ in the north and up directions, respectively, compared with $0.121 \mathrm{~m}$ and $0.224 \mathrm{~m}$ for the GRC-PPP. The GE-PPP solution's accuracy is improved from $0.205 \mathrm{~m}$ in the east direction to $0.189 \mathrm{~m}$ for the GRC-PPP solution. The GR-PPP, GE-PPP, GRC-PPP, and GRE-PPP solutions show similar levels of positioning accuracy in the up direction. Additionally, the GR-PPP solution's accuracy is improved from $0.199 \mathrm{~m}$ and $0.183 \mathrm{~m}$ in the east and north directions, respectively, to $0.189 \mathrm{~m}$ and $0.121 \mathrm{~m}$ for the GRC-PPP solution. Although this presents only a slight improvement in the east direction, it shows an improvement of $34 \%$ in the north direction. The GRE-PPP accuracy is improved by $25 \%$, $52 \%$, and $27 \%$ in the east, north, and up directions, respectively, compared with the GRECPPP solution. Moreover, the GRC-PPP solution is improved by $23 \%, 30 \%$, and $24 \%$ in the 
east, north, and up directions, respectively, compared with the GREC-PPP solution. In general, decimeter-level positioning accuracy in both east and up directions and centimeterlevel positioning accuracy in the north direction is achieved through the IRKF-based quadconstellation GNSS PPP.

Table 3. RMS error and mean error $(\mathrm{m})$ in the east, north, and up directions for real-time GR-PPP, GE-PPP, GRC-PPP, GRE-PPP, and GREC-PPP-static field trial

\begin{tabular}{|c|c|c|c|c|c|c|c|c|c|c|}
\hline \multirow{2}{*}{ Directions } & \multicolumn{2}{|c|}{ GR-PPP } & \multicolumn{2}{c|}{ GE-PPP } & \multicolumn{2}{c|}{ GRC-PPP } & \multicolumn{2}{c|}{ GRE-PPP } & \multicolumn{2}{c|}{ GREC-PPP } \\
\cline { 2 - 11 } & $\boldsymbol{R M S}$ & Mean & $\boldsymbol{R M S}$ & Mean & $\boldsymbol{R M S}$ & Mean & $\boldsymbol{R M S}$ & Mean & $\boldsymbol{R} M \boldsymbol{M}$ & Mean \\
\hline East & 0.199 & 0.163 & 0.205 & 0.119 & 0.189 & 0.153 & 0.197 & 0.116 & 0.147 & 0.111 \\
\hline North & 0.183 & 0.076 & 0.121 & 0.071 & 0.121 & 0.072 & 0.177 & 0.087 & 0.085 & 0.089 \\
\hline Up & 0.231 & 0.181 & 0.237 & 0.124 & 0.224 & 0.184 & 0.234 & 0.139 & 0.171 & 0.167 \\
\hline
\end{tabular}

\subsection{Simulated-kine matic field trial}

The field trial was conducted in a suburban area with an open-sky environment on June 20, 2020, and this lasted for 2 hours. Figure 8 shows the visibility of the visible GNSS satellites during the whole data collection period. The minimum number of satellites was six, the maximum number was 25 , and the elevation mask angle was $7.5^{\circ}$. The process noise for the receiver's position was adjusted to mimic the kinematic processing to assess the positioning performance in a simulated-kinematic mode under an open-sky environment. Additionally, the filter was forced to restart after 1 hour to evaluate the quad-constellation GNSS PPP's reconvergence performance. The positioning errors of the real-time GR-PPP, GE-PPP, GRCPPP, GRE-PPP, and GREC-PPP solutions through the IRKF in the east, north, and up directions, respectively, are presented in Figures 8-11. As can be seen, the GREC-PPP and GRE-PPP solutions show similar performances in the first hour of the data set in the east and north directions. However, the GREC-PPP solution shows better performance than the GREPPP solution in the up direction. The GE-PPP solution shows the worst performance in the east and north directions, but it shows a performance comparable to that of the GRE-PPP solution in the up direction. At the start of the second hour, the GREC-PPP shows the best convergence in the north and up directions and with superiority of the GE-PPP solution in the east direction. 


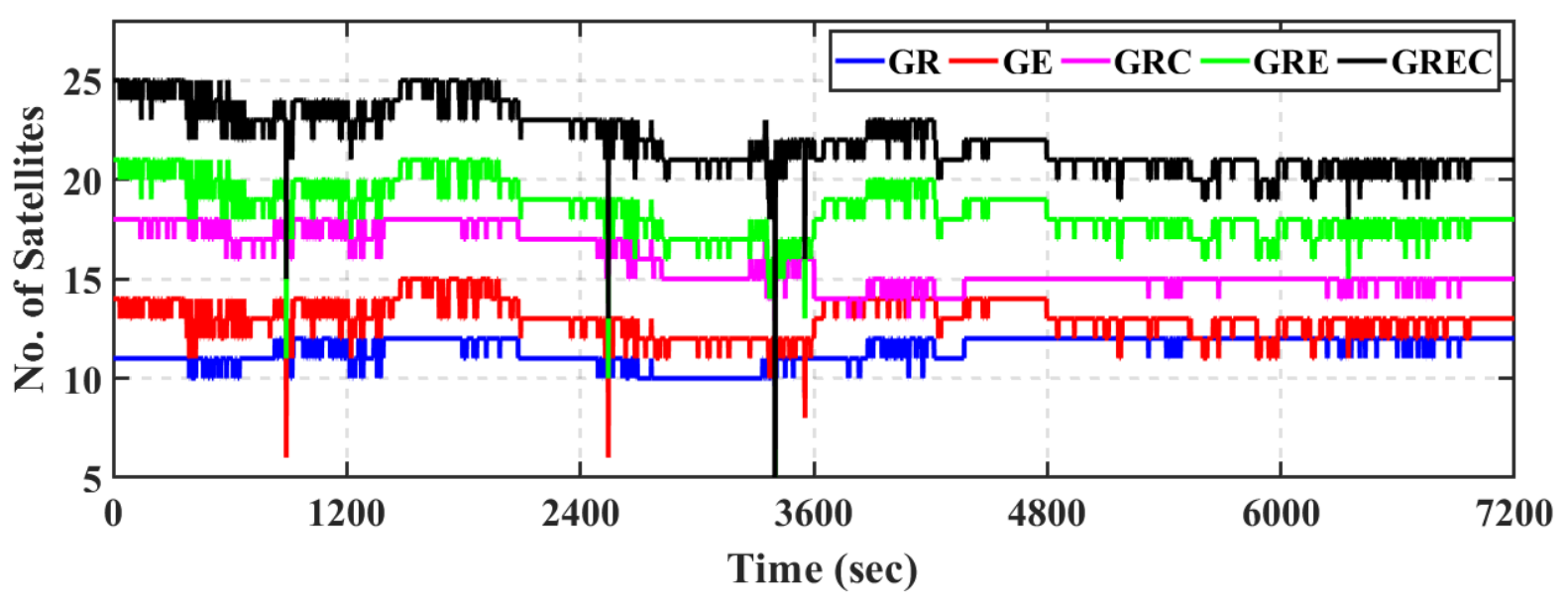

Figure 8. Simulated-kinematic field trial: number of observable GPS + GLONASS, GPS + Galileo, GPS + GLONASS + BeiDou, GPS + GLONASS + Galileo, and GPS + GLONASS + Galileo + BeiDou satellites

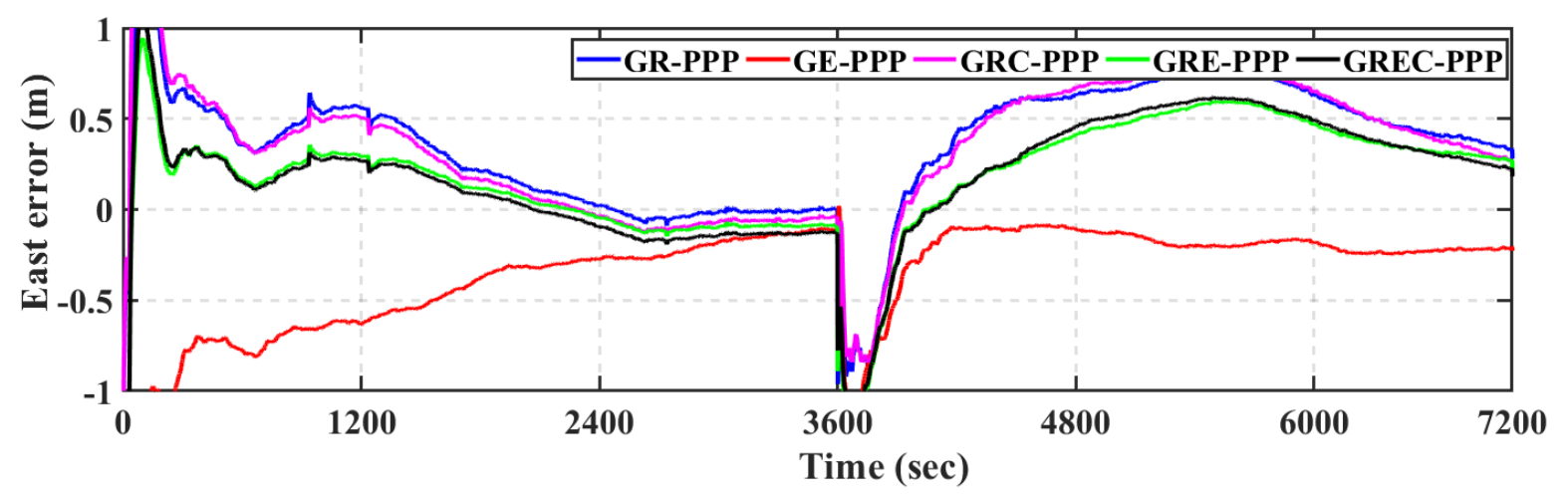

Figure 9. Simulated-kinematic field trial: east positioning error of real-time GR-PPP, GE-PPP, GRE-PPP, GRC-PPP, and GREC-PPP solutions

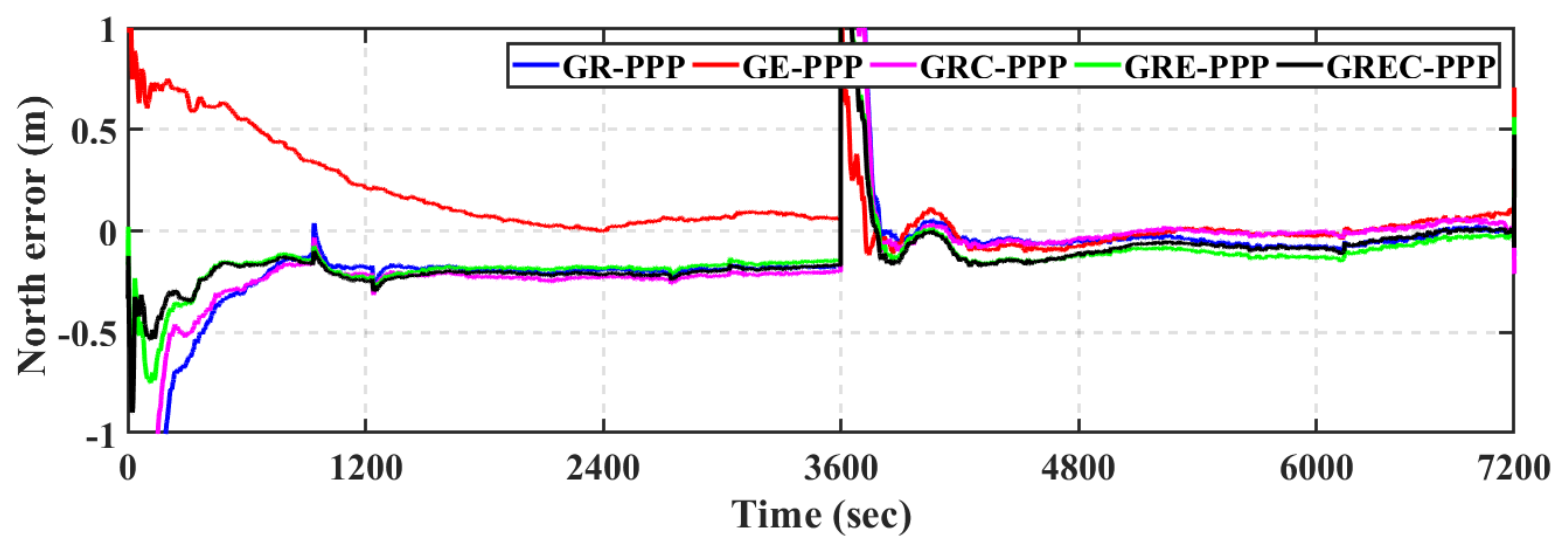

Figure 10. Simulated-kinematic field trial: north positioning error of real-time GR-PPP, GE-PPP, GRE-PPP, GRC-PPP, and GREC-PPP solutions 


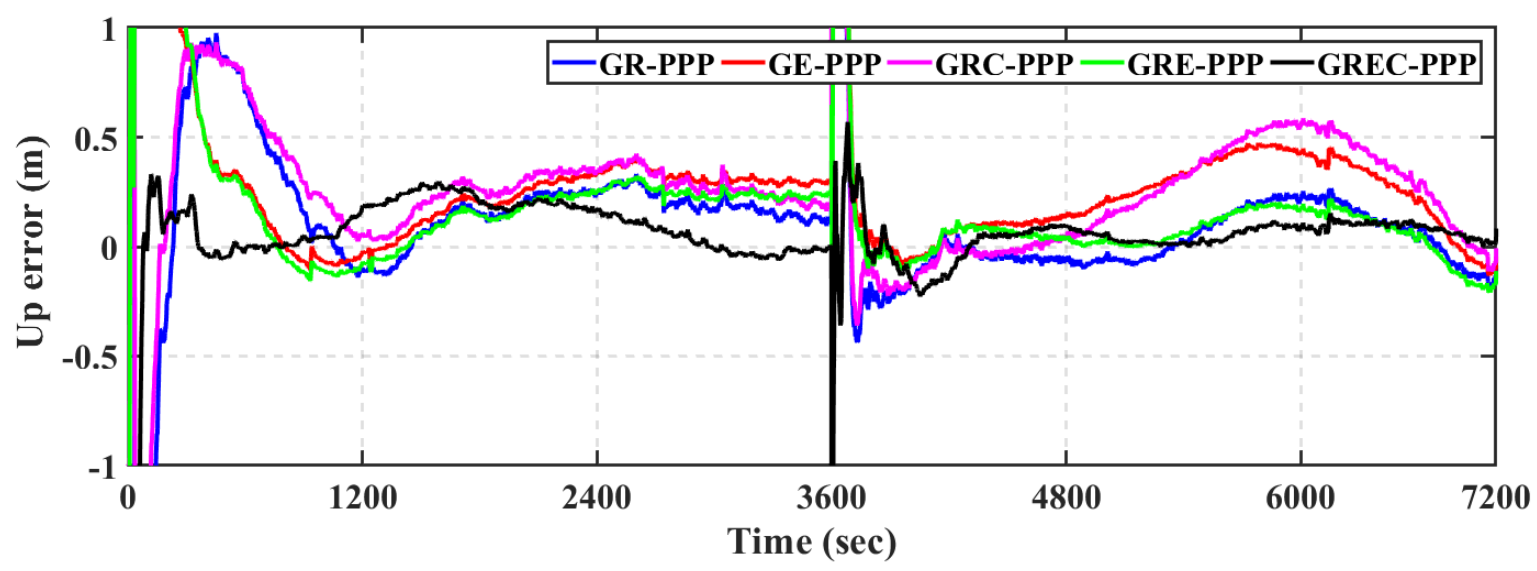

Figure 11. Simulated-kinematic field trial: up positioning error of real-time GR-PPP, GE-PPP, GRE-PPP, GRC-PPP, and GREC-PPP solutions

Another representation of the GR-PPP, GE-PPP, GRC-PPP, GRE-PPP, and GREC-PPP solutions' errors is shown in Figure 12. In this representation, the cumulative distribution function (CDF) of the horizontal and vertical positioning errors is determined to assess the quad-constellation GNSS PPP solution. As shown in Figure 12, about $95 \%$ of the horizontal positioning errors of the GREC-PPP solution are $<0.5 \mathrm{~m}$, and about $98 \%$ of the vertical positioning errors are $<0.5 \mathrm{~m}$. Additionally, the GRE-PPP solution shows comparable performance to the GREC-PPP solution in the horizontal direction. For the GE-PPP, GRPPP, and GRC-PPP solutions, about $95 \%$ of the horizontal positioning errors are $<1 \mathrm{~m}$, and $98 \%$ of the vertical positioning errors are $<1.5 \mathrm{~m}$. This represents a significant improvement in the positioning performance through the quad-constellation GNSS PPP. The CDF confirms that both the GREC-PPP and the GRE-PPP solutions show similar performance in the horizontal direction and outperform the GR-PPP, GE-PPP, and GRC-PPP solutions. Moreover, the GREC-PPP solution shows the best performance in the vertical direction.
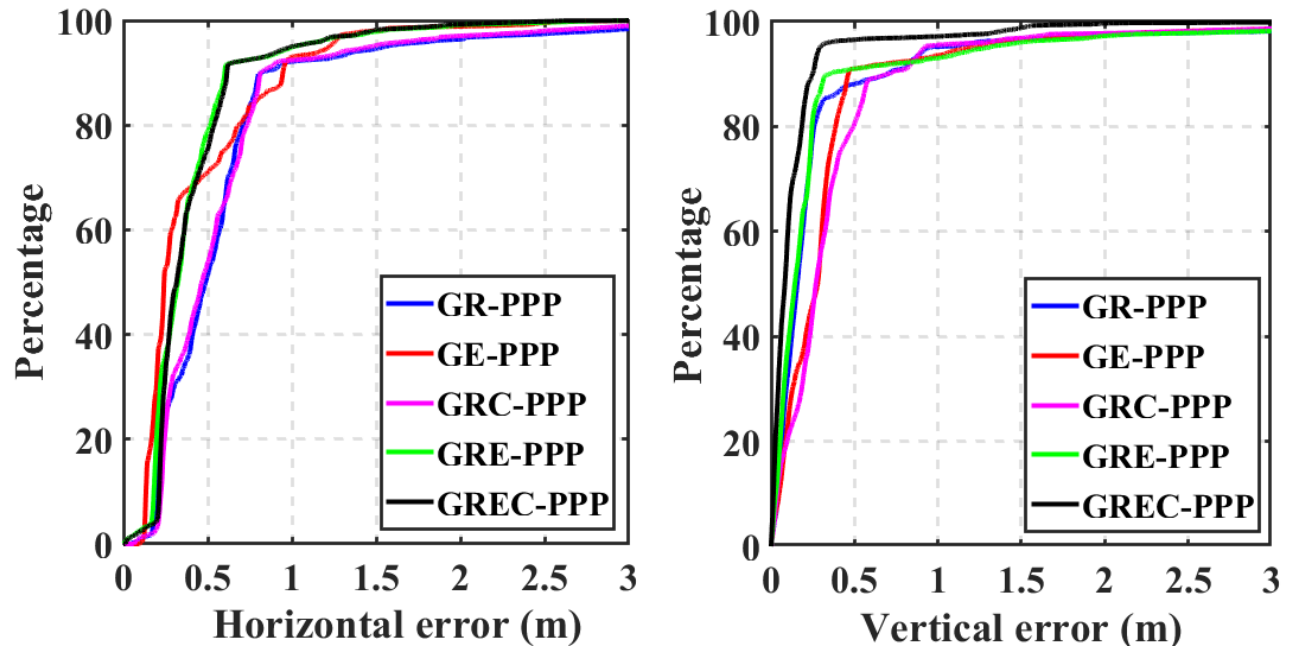

Figure 12. CDF for horizontal error (left) and vertical error (right) of real-time GR-PPP, GE-PPP, GRC-PPP, GRE-PPP, and GREC-PPP solutions-simulated-kinematic field trial

To assess the overall positioning accuracy, the RMS and mean of the positioning errors are presented in Table 4. The RMS and mean are estimated for the positioning errors within the 2hour data session. It can be seen that the positioning accuracy of the GREC-PPP solution is at the decimeter level in all positioning components. The accuracy of the GRE-PPP solution in 
the up direction is improved from $0.767 \mathrm{~m}$ to $0.337 \mathrm{~m}$ for the GREC-PPP solution. The accuracy of the GRC-PPP solution is improved by $31 \%, 40 \%$, and $51 \%$ in the east, north, and up directions, respectively, compared with the GREC-PPP solution. The accuracy of the GEPPP solution is improved from $0.506 \mathrm{~m}, 0.483 \mathrm{~m}$, and $0.767 \mathrm{~m}$ in the east, north, and up directions, respectively, to $0.414 \mathrm{~m}, 0.309 \mathrm{~m}$, and $0.337 \mathrm{~m}$ for the GREC-PPP solution. Moreover, the GR-PPP solution is significantly improved by $30 \%, 55 \%$, and $60 \%$ in the east, north, and up directions, respectively, compared with the GREC-PPP solution. Meanwhile, the GRC-PPP solution shows similar positioning accuracy as the GE-PPP solution and is slightly better than the GR-PPP solution. This is a result of the low number of visible BeiDou satellites. On the other hand, the accuracy of the GRE-PPP solution is better than that of both the GE-PPP and GR-PPP solutions due to the high visibility of GPS, GLONASS, and Galileo satellites, as shown in Figure 8.

Table 4. RMS error and mean error $(\mathrm{m})$ in the east, north, and up directions of real-time GR-PPP, GE-PPP, GRB-PPP, GRE-PPP, and GREB-PPP-simulated kinematic field trial

\begin{tabular}{|c|c|c|c|c|c|c|c|c|c|c|}
\hline \multirow{2}{*}{ Directions } & \multicolumn{2}{|c|}{ GR-PPP } & \multicolumn{2}{c|}{ GE-PPP } & \multicolumn{2}{c|}{ GRC-PPP } & \multicolumn{2}{c|}{ GRE-PPP } & \multicolumn{2}{c|}{ GREC-PPP } \\
\cline { 2 - 11 } & $\boldsymbol{R M S}$ & Mean & $\boldsymbol{R M S}$ & Mean & $\boldsymbol{R M S}$ & Mean & $\boldsymbol{R M S}$ & Mean & $\boldsymbol{R M S}$ & Mean \\
\hline East & 0.587 & 0.459 & 0.506 & 0.378 & 0.598 & 0.462 & 0.406 & 0.317 & 0.414 & 0.327 \\
\hline North & 0.685 & 0.266 & 0.483 & 0.158 & 0.513 & 0.234 & 0.325 & 0.197 & 0.309 & 0.192 \\
\hline Up & 0.853 & 0.327 & 0.767 & 0.359 & 0.701 & 0.388 & 0.670 & 0.305 & 0.337 & 0.145 \\
\hline
\end{tabular}

\subsection{Kine matic field trial}

The field trial was conducted in an urban area with a challenging environment that included tall buildings and trees near the road (about $3 \mathrm{~m}$ ). The trial was conducted on June 22, 2020, and it lasted for about 33 minutes. The trajectory of the field trial is shown in Figure 13. The $\mathrm{u}$-blox patch antenna is mounted on the floor of the vehicle. GPS, GLONASS, and Galileo measurements were logged using a laptop connected to the u-blox ZED-F9P GNSS module. As shown in Figure 14, the minimum number of satellites was $<5$, and the maximum number was 21. Figures 15-17 show the convergence performances of the real-time GR-PPP, GEPPP, and GRE-PPP solutions through the IRKF for all positioning components. As can be seen, the GR-PPP solution presents the worst positioning performance compared to both the GE-PPP and GRE-PPP solutions. This is due to the low number of visible GLONASS satellites during the field trial data collection. Both the GE-PPP and GRE-PPP solutions show similar performances in the first 3 minutes. However, with the passage of time, the GRE-PPP solution shows better performance than the GE-PPP counterpart. The majority of the positioning error spikes are smoothed through the triple-constellation GNSS PPP, leading to a more stable positioning solution. Additionally, the positioning solutions are degraded with time, especially in the up directions, due to the high variability of the satellites. 


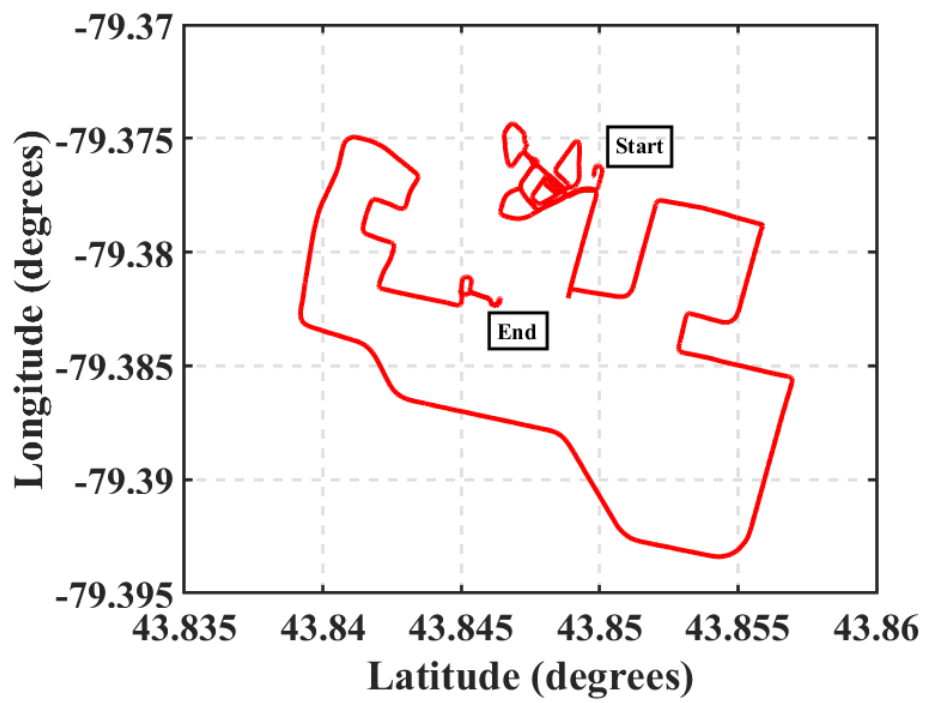

Figure 13. Trajectory of the kinematic field trial: Toronto, Ontario, Canada (June 22, 2020)

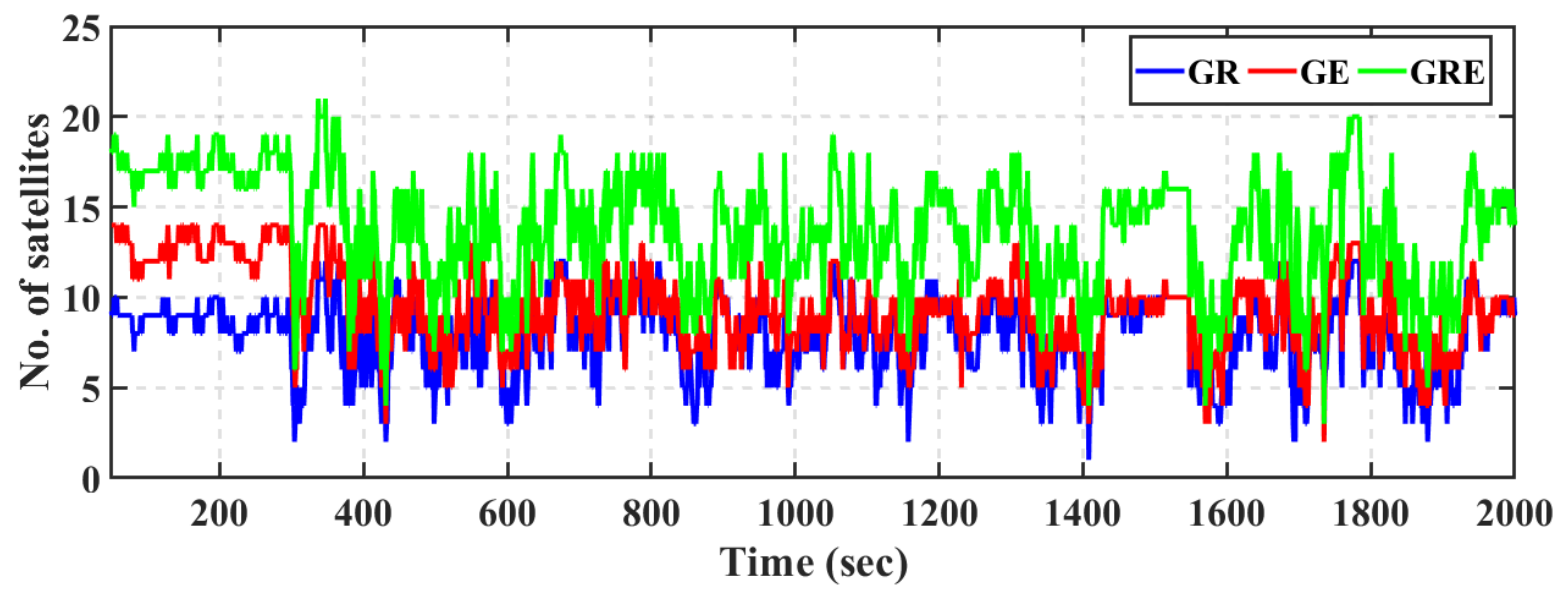

Figure 14. Kinematic field trial: number of observable GPS + GLONASS, GPS + Galileo, GPS + GLONASS + Galileo satellites

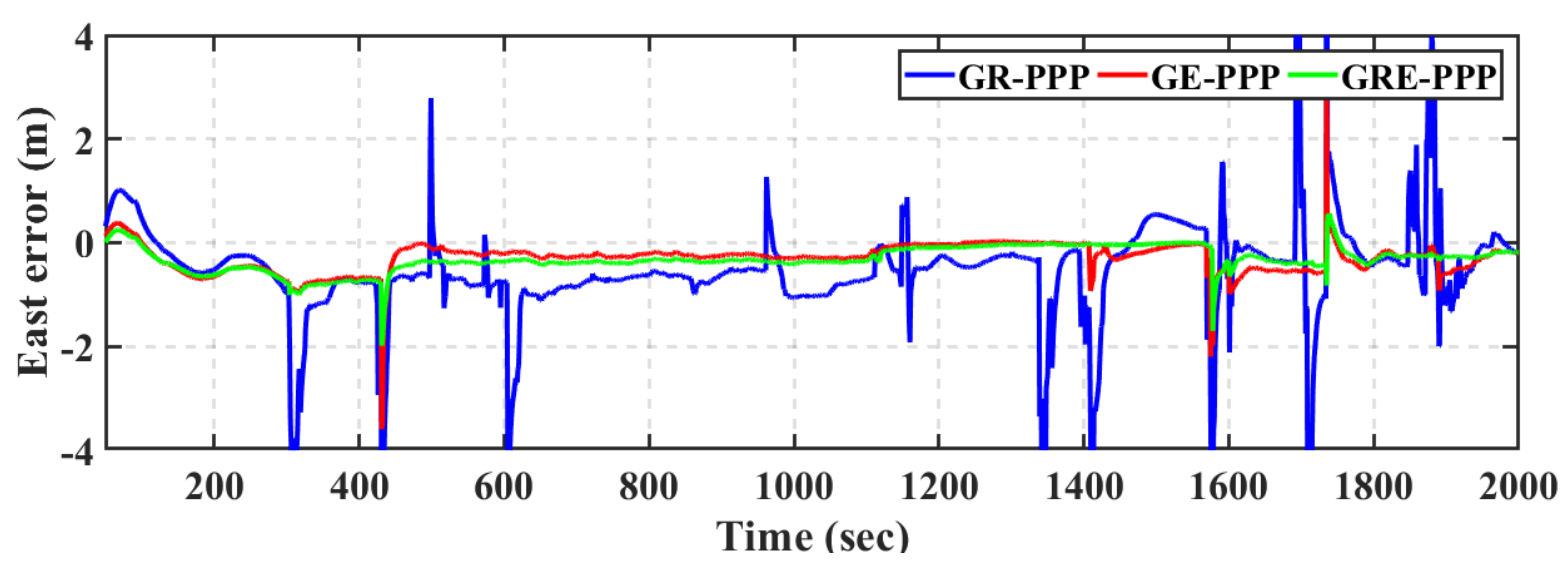

Figure 15. Kinematic field trial: east positioning error of real-time GR-PPP, GE-PPP, and GRE-PPP solutions 


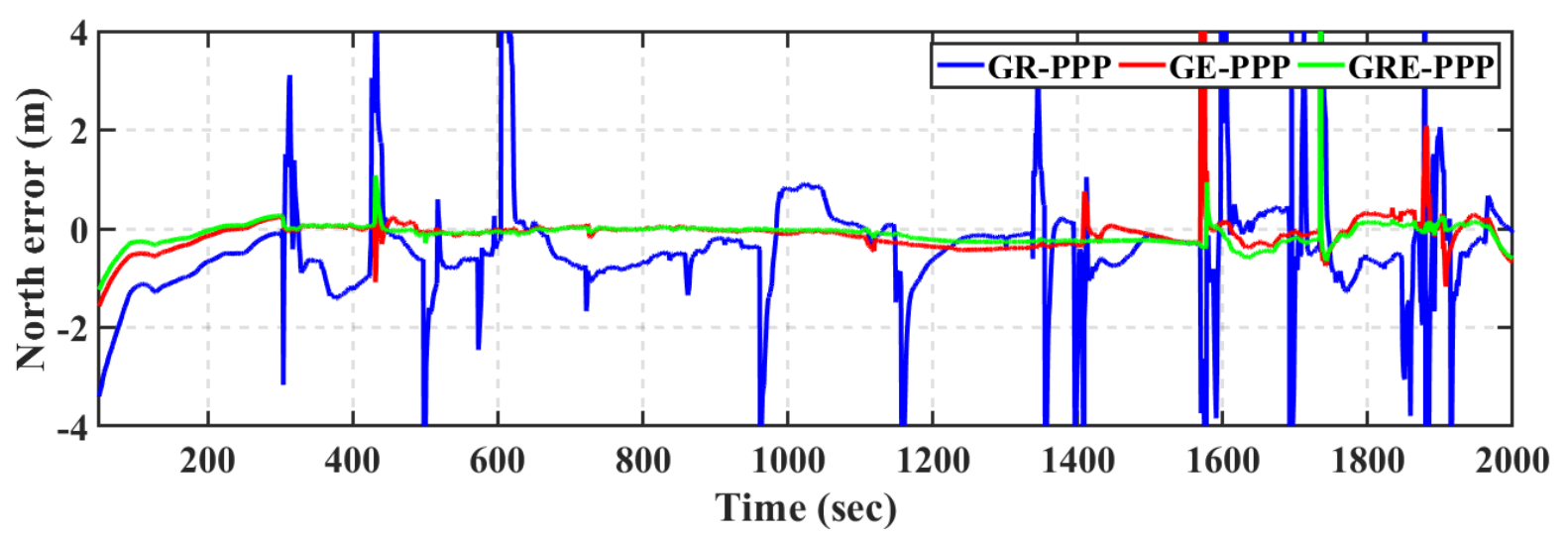

Figure 16. Kinematic field trial: north positioning error of real-time GR-PPP, GE-PPP, and GREPPP solutions

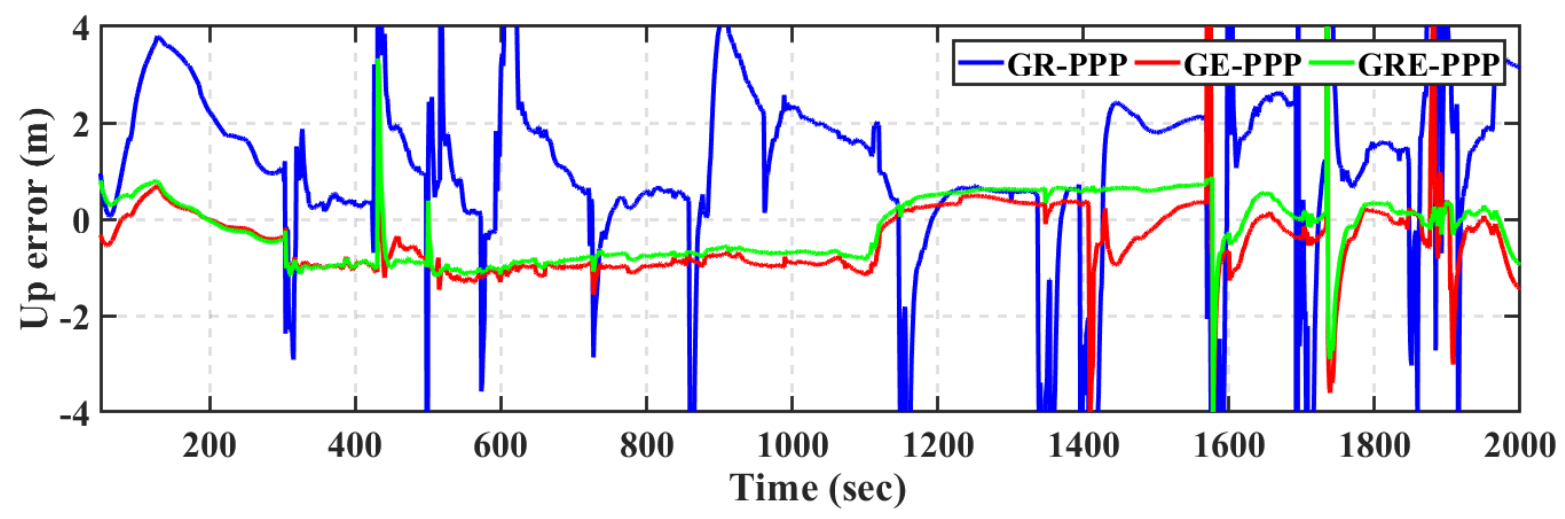

Figure 17. Kinematic field trial: up positioning error of real-time GR-PPP, GE-PPP, and GRE-PPP solutions

To further assess the triple-constellation GNSS PPP through the IRKF, both the RMS and mean for each positioning component of the GR-PPP, GE-PPP, and GRE-PPP solutions are presented in Table 5. As shown in the table, the GR-PPP solution shows the worst performance, which coincides with Figures 15-17. The positioning accuracy is at the meter level in both the horizontal and vertical directions. Meanwhile, the GE-PPP solution shows much better positioning accuracy than the GR-PPP counterpart. The accuracy of the GR-PPP solution is improved from $1.224 \mathrm{~m}, 1.877 \mathrm{~m}$, and $3.056 \mathrm{~m}$ in the east, north, and up directions, respectively, to $0.409 \mathrm{~m}, 0.503 \mathrm{~m}$, and $1.165 \mathrm{~m}$ for the GE-PPP counterpart. Additionally, the accuracy of the GE-PPP solution is improved from $0.409 \mathrm{~m}, 0.503 \mathrm{~m}$, and $1.165 \mathrm{~m}$ in the east, north, and up directions, respectively, to $0.390 \mathrm{~m}, 0.308 \mathrm{~m}$, and $0.729 \mathrm{~m}$ for the GREPPP solution. This represents an improvement of 5\%,38\%, and $37 \%$ in the east, north, and up directions, respectively. The overall positioning accuracy of the GRE-PPP solution is at the decimeter and submeter levels in the horizontal and vertical directions, respectively. This is worse than the static and the simulated-kinematic trials, which is mainly due to the low number of visible satellites during the kinematic field trial. 
Table 5. RMS error and mean error $(\mathrm{m})$ in the east, north, and up directions of real-time GR-PPP, GE-PPP, and GRE-PPP-kinematic field trial

\begin{tabular}{|c|c|c|c|c|c|c|}
\hline \multirow{2}{*}{ Directions } & \multicolumn{2}{|c|}{ GR-PPP } & \multicolumn{2}{c|}{ GE-PPP } & \multicolumn{2}{c|}{ GRE-PPP } \\
\cline { 2 - 7 } & $\boldsymbol{R M S}$ & Mean & $\boldsymbol{R M S}$ & Mean & $\boldsymbol{R M S}$ & Mean \\
\hline East & 1.224 & 0.785 & 0.409 & 0.308 & 0.390 & 0.324 \\
\hline North & 1.877 & 0.876 & 0.503 & 0.212 & 0.308 & 0.160 \\
\hline Up & 3.056 & 1.959 & 1.165 & 0.677 & 0.729 & 0.611 \\
\hline
\end{tabular}

\section{CONCLUSIONS}

In this study, a real-time quad-constellation GNSS PPP using the low-cost u-blox Z9D-F9P module was thoroughly assessed. The PPP solution was assessed in static and simulatedkinematic modes under an open-sky environment and in an actual kinematic mode under a challenging environment. The IRKF was used as the estimation filter, and the measurements were processed in different modes, namely, real-time GPS + GLONASS PPP (GR-PPP), GPS + Galileo PPP (GE-PPP), GPS + GLONASS + Galileo PPP (GRE-PPP), GPS + GLONASS + BeiDou PPP (GRC-PPP), and GPS + GLONASS + Galileo + BeiDou PPP (GREC) modes. In the static mode, the accuracy of the GRE-PPP and GRC-PPP solutions was significantly improved compared with the GREC PPP solution. The GRECPPP accuracy was at the centimeter level in the north direction and at the decimeter level in both east and up directions. The GREC PPP further enhanced the solution convergence compared with the GRE-PPP and GRC-PPP counterparts. In the simulated-kinematic mode, the GREC-PPP showed the best convergence for all positioning components. Additionally, the positioning accuracy of both GRE-PPP and GRC-PPP solutions was improved from the decimeter and submeter levels in the horizontal and vertical components, respectively, to be at the decimeter level for the GREC-PPP solution. Under a challenging environment in which the satellite number was highly variable, the GRE-PPP improved the positioning accuracy and enhanced the solution convergence compared with the GR-PPP and GE-PPP counterparts. Moreover, the accuracy of the GR-PPP solution was improved from the meter level in the horizontal and vertical directions, respectively, to the decimeter and submeter levels for the GRE-PPP solution. Moreover, the GE-PPP's accuracy was improved by 5\%, $38 \%$, and $37 \%$ in the east, north, and up directions, respectively, compared with the GREPPP.

\section{REFERENCES}

Böhm J., Möller G., Schindelegger M., Pain G., Weber R. (2015) Development of an improved empirical model for slant delays in the troposphere (GPT2w), GPS Solutions, Vol. 19, 433-441.

Dan S., Santra A., Mahato S., Bose A. (2020) On use of low cost, compact GNSS modules for ionosphere monitoring.

De Bakker P.F., Tiberius C.C. (2017) Real-time multi-GNSS single-frequency precise point positioning, GPS Solutions, Vol. 21, 1791-1803.

Elmezayen A., El-Rabbany A. (2019) Precise point positioning using world's first dualfrequency GPS/Galileo Smartphone, Sensors, Vol. 19, 2593. 
Elsheikh M., Yang H., Nie Z., Liu F., Gao Y. (2018) Testing and analysis of instant PPP using freely available augmentation corrections, Proceedings of the 31st International Technical Meeting of the Satellite Division of the Institute of Navigation (ION GNSS + 2018), Miami, FL, USA, 2018, 24-28.

Elsobeiey M., Al-Harbi S. (2016) Performance of real-time precise point positioning using IGS real-time service, GPS Solutions, Vol. 20, 565-571.

IGS. (2020) International GNSS real-time service, Available at: http:/www.igs.org/rts [Accessed May 25, 2020].

Kouba J. (2015) A guide to using international GNSS service (IGS) products, IGS [Online]. Available at: https://kb.igs.org/hc/en-us/articles/201271873-A-Guide-to-Using-the-IGSProducts [Accessed December 25, 2019].

Krietemeyer A., Marel H.V.D., Giesen N.V.D., Veldhuis M.-C.T. (2020) High quality zenith tropospheric delay estimation using a low-cost dual-frequency receiver and relative antenna calibration. Remote Sensing, Vol. 12, 1393.

Nie Z., Liu, F., Gao, Y. (2020) Real-time precise point positioning with a low-cost dualfrequency GNSS device, GPS Solutions, Vol. 24, 9.

Nie Z., Yang H., Zhou P., Gao Y., Wang Z. (2019) Quality assessment of CNES real-time ionospheric products, GPS Solutions, Vol. 23, 11.

Psychas D., Bruno J., Massarweh L., Darugna F. (2019) Towards sub-meter positioning using android raw GNSS measurements, 32nd International Technical Meeting of the Satellite Division of the Institute of Navigation, ION GNSS+ 2019, 2019, Institute of Navigation, 3917-3931.

Rizos C., Montenbruck O., Weber R., Weber G., Neilan R., Hugentobler U. (2013) The IGS MGEX experiment as a milestone for a comprehensive multi-GNSS service, Proceedings of ION PNT, Honolulu, Hawaii 289-295.

Saastamoinen. (1973) Contributions to the theory of atmospheric refraction, Bulletin Géodésique, Vol. 107, 13-34.

U-Blox. (2020) ZED-F9P module Available at: https://www.u-blox.com/en/product/zed-f9pmodule [Accessed May 30, 2020].

US-Coast-Guard. GPS constellation status. Available at: https://www.navcen.uscg.gov/?Do=constellationStatus [Accessed January 21, 2020].

Wang Z., Li Z., Wang L., Wang X., Yuan H. (2018) Assessment of multiple GNSS real-time SSR products from different analysis centers, ISPRS International Journal of GeoInformation, Vol. 7, 85.

Wu Q., Sun M., Zhou C., Zhang P. (2019) Precise point positioning using dual-frequency GNSS observations on smartphone, Sensors, Vol. 19, 2189.

$\mathrm{Xu}, \mathrm{G} ., \mathrm{Xu}, \mathrm{Y}$. (2016) GPS: Theory, algorithms and applications, Springer: Berlin/Heidelberg, Germany.

Zhang Q., Zhao L., Zhao L., Zhou J. (2018) An improved robust adaptive Kalman filter for GNSS precise point positioning, IEEE Sensors Journal, Vol. 18, 4176-4186. 
Received: 2021-04-01

Reviewed: 2021-05-21 (D. Próchniewicz) and 2021-06-14 (M. Grzegorzewski)

Accepted: 2021-07-03 